\title{
The Earth is Our Common Home
}

\author{
V. V. Goncharuk, Academician of National Academy of Science of Ukraine, Professor \\ Dumanskii Institute of Colloid and Water Chemistry, \\ National Academy of Sciences of Ukraine, Kyiv \\ e-mail: ghonch@iccwc.kiev.ua
}

DOI: $10.3103 / \mathrm{S} 1063455 \mathrm{X} 19050011$

For thousands of years, man has been mastering the planet Earth by using all the natural resources including water, air, land, and minerals for the vital activity of mankind. At present, more than 7 billion people inhabit our planet, and the ever-growing resources are required to accommodate their needs. The supercritical situation has arisen with the renewal and restoration of the human environment, and primarily with the preservation of high quality and genetically safe sources of drinking water. I submit that it is the water basin of the planet that constitutes the main "immune system" of the biosphere. This powerful buffer zone has resisted and neutralized all technogenic loads originated by the economic activity of man. However, even now, at the beginning of the XXI century, it can be stated that the planet, its whole ecosystem, and biosphere, including the multitude of the whole aggregate of organisms, especially of microflora inhabiting our plant are in the state of world technogenic transformation of enormous scale.

The tremendous development of economy, nuclear power engineering, space exploration, and also militarization of society have led to the ecological catastrophe in the biosphere, ionosphere, and stratosphere, and their transformation into technosphere. This is testified by intense changes in the world of flora and fauna, extinction of different species of plants and animals, the appearance of genetically modified lifeforms, and the total immunodeficiency in humans.

The planet under the name of Earth can be considered as a huge space vehicle traveling in space, while we as astronauts explore the near and far outer space having quite limited resources and our natural life-support systems. In practical terms, we have lost the high-qualitynatural freshwater suitable for drinking. Today about 100 million anthropogenic compounds artificially generated by humans circulate in the environment. They include also toxic chemicals such as pesticides and defoliants, surfactants, pharmaceuticals, and a variety of other chemicals earlier unknown on the Earth that mostly are not degraded in natural conditions.

All this took its toll on our biosphere. In the process of adapting and adjusting to new living conditions, the biota changes itself, mutates, and acquires new earlier unknown forms and properties that could be generally harmful to humans.

The use of any chemical agents for the disinfection of water results in the appearance of newresistant forms of microflora that could possess more toxic properties than the initial ones for humans and other biological entities. We are observing the evolution of infectious diseases that do not respond to medical treatment by traditional methods. Increasingly frequently, the water becomes a dangerous source of infectious diseases with unpredictable etiology. Moreover, we are witnessing a wide and fast spread of resistant and adapted to disinfectants new forms of microorganisms that, even in strongly chlorinated water, acquire new properties manifested in their nonculturability, i.e., bacteria are in a state of very low metabolic activity and do not divide but are alive for a long time. This phenomenon sets a new challenge for microbiologists aimed at searching for new methods of assessing microorganism's viability.

Thus, the rising anthropogenic impact on the environment and the progressive and irreversible impact on different forms of life requires a new approach toward the quality assessment of drinking water as the resource most important for man. We propose a fundamentally new standard for drinking water that is based on the assessment of acute and chronic toxicity and genotoxicity at the organismic and cellular levels of freshwater organisms. All these approaches and control methods are described in detail in the monograph by Vladislav V. Goncharuk, Drinking Water. Physics, Chemistry and Biology (Springer, 2014) and in the National Standard of Ukraine: DRINKING WATER. Requirements and quality control methods (DSTU 7525:2014).

Since life is the cellular form of matter, human health depends on the vital activity of every cell. 